\title{
Étude numérique pour l'optimisation de la réhabilitation d'ouvrages d'assainissement à l'aide de composites
}

\author{
Stephan Kesteloot $^{1,3, a}$, Chafika Djelal ${ }^{1},{\text { SaÏd } \text { Baraka }^{2} \text { et Idriss Benslimane }}^{3}$ \\ ${ }^{1}$ Laboratoire Artois Mécanique et Habitat, Équipe Matériaux, Thermiques et Instrumentations, IUT, Rue de l'Université, \\ 62400 Béthune, France \\ 2 3Abtp, Auscultations, Analyses et Avaries, 17-25 rue Charles Graindorge, 93170 Bagnolet, France \\ 3 Structure \& Réhabilitation, 72-74 avenue Gambetta, 93170 Bagnolet, France
}

Reçu le 16 janvier 2004, accepté le 9 septembre 2004

\begin{abstract}
Résumé - Les réseaux d'assainissement subissent de nombreuses dégradations d'ordres structurelles. Les maîtres d'ouvrages estiment à $10 \%$ du linéaire au moins, la longueur des collecteurs dont l'état de dégradation structurelle exige des travaux. Cet article concerne la restructuration d'ouvrages d'assainissement par lamelles composites. Afin d'appliquer cette technique de renforcement, une analyse par éléments finis est nécessaire pour connaître le mécanisme de ruine et donc les zones à renforcer. Une campagne expérimentale a permis de caractériser les différents matériaux (béton, colle et lamelles de carbone) et d'obtenir leurs lois de comportement. Une comparaison entre ovoïde témoin et ovoïde renforcé par lamelle de carbone pourra être effectuée.
\end{abstract}

Mots clés : Composites / béton / colle / éléments finis / ouvrages d'assainissement / théorie de la fissuration / taux de restitution d'énergie / réparation / renforcement

\begin{abstract}
Numerical study for the optimization of the rehabilitation of sewerages systems using composites. Sewerage systems undergo various structural damages. It is estimated that $10 \%$ of the total length need rehabilitation. This publication is concerned with restructuring by carbon fiber plates. The Finite Element Method is necessary in order to capture the collapse mecanism and therefore to locate the zones to strengthen. Experimental testing has been conducted to characterize the different materials (concrete, glue, and carbon fibers plates) and then obtain their constitutive laws. A comparison between witness samples and carbon reinforced ovoids (egg-shaped sewers) can be done.
\end{abstract}

Key words: Composite / concrete / glue / finite elements / sewerage structures / theory of cracking / rate of energy restitution / repair / reinforcements

\section{Introduction}

C'est au début des années 1980, qu'en France, de nombreux maîtres d'ouvrages ont pris conscience de l'importance du patrimoine que représentent les réseaux d'assainissement. En effet, ces ouvrages datent en grande majorité de la fin du XIX ${ }^{\mathrm{e}}$ siècle, début du XX $\mathrm{XX}^{\mathrm{e}}$. Or l'urbanisation, la position stratégique de ces ouvrages et l'encombrement des sous-sols sont autant d'éléments qui rendent impossibles ou tout au moins très difficiles et très onéreuses des reconstructions ou des constructions neuves. Par ailleurs ces ouvrages ont, en règle générale

\footnotetext{
a Auteur correspondant :

stephan.kesteloot@fsa.univ-artois.fr
}

été surdimensionnés lors de leur réalisation et ils offrent encore à ce jour des capacités hydrauliques suffisantes [1] .

Il s'en suit que d'une époque de reconstruction et de construction, nous sommes passés progressivement à l'aire de la réhabilitation. Ainsi pour renforcer ou pour réparer ces ouvrages, de nombreuses techniques utilisant divers produits et divers procédés ont vu le jour.

Une distinction doit être réalisée entre les réhabilitations susceptibles d'être structurantes, c'est-àdire susceptibles de renforcer un ouvrage endommagé ou soumis ultérieurement à des surcharges pour lesquelles il n'a pas été dimensionné et les réhabilitations qui n'ont pas d'autre but que d'étancher ou de protéger les structures existantes. 


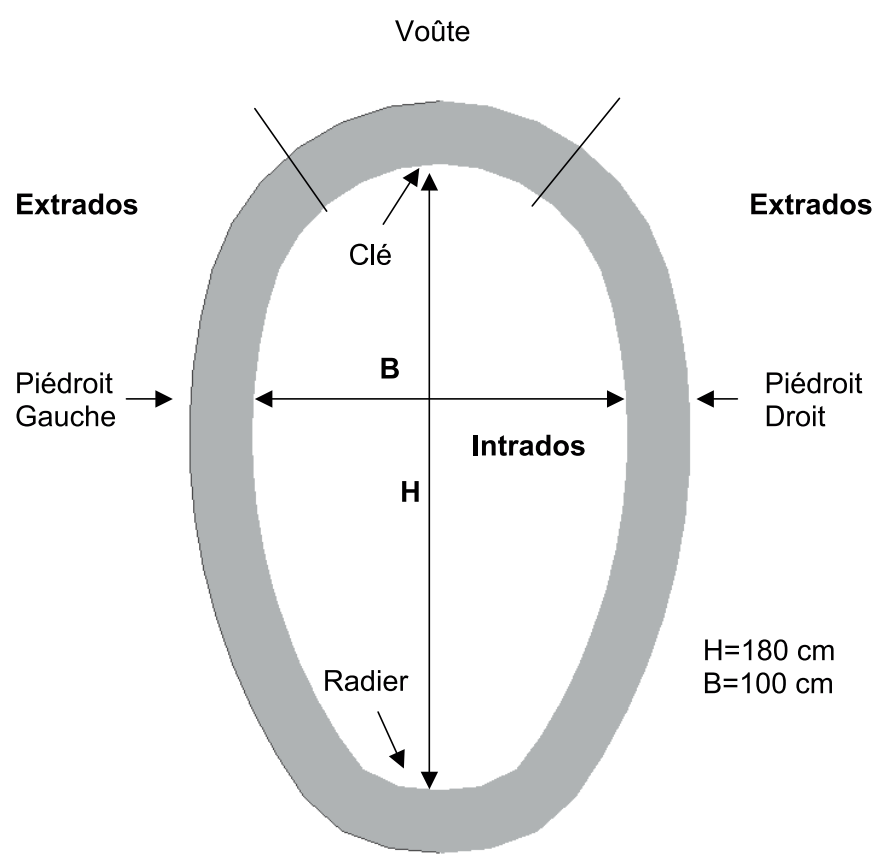

Fig. 1. Schéma d'un ovoïde T180.

Les réseaux d'assainissement sont de différentes natures, ils peuvent être visitables ou non et sont constitués de différents matériaux (maçonnerie, béton).

Leurs formes sont variables, ils sont circulaires ou ovoïdaux. Cette étude s'intéresse principalement aux réseaux d'assainissement en béton armé de forme ovoïdale (T180).

Les ovoïdes (Fig. 1) sont constitués d'une voûte, de piédroits et d'un radier.

Les réseaux d'assainissement visitables subissent de nombreuses dégradations [2] pouvant provenir de l'intérieur ou de l'extérieur de l'ouvrage. De par leur fonction, ils sont soit soumis à des attaques chimiques $\left(\mathrm{H}_{2} \mathrm{~S}\right)$ [3], soit à des dégradations superficielles ou structurelles :

- tassement du terrain environnant,

- surcharges à la surface du sol,

- erreurs de conception.

Les dégradations peuvent se situer dans des endroits particuliers ou sur l'ensemble de l'ouvrage, c'est-à-dire de la voûte au radier en passant par les piédroits, à l'intrados comme à l'extrados.

Afin de rendre les fonctions primaires d'un ouvrage dégradé, il existe de nombreuses techniques de réparation et de renforcement, mais elles sont à la fois coûteuses et réparties sur la périphérie de l'ouvrage (injection, chemisage par projection de béton ou mortier, tubage par élément préfabriqué) [2].

Dans le domaine du bâtiment et des travaux publics, les méthodes de renforcement ponctuelles à l'aide de matériaux composites [4] sont maintenant bien maîtrisées. Ces matériaux se présentent sous forme de plaques pultrudées ou de tissus.
Les Sociétés Sika et Structure \& Réhabilitation en collaboration avec le laboratoire LAMH se sont données pour objectif d'appliquer cette technique aux ouvrages d'assainissement grâce à un vaste programme expérimental et des vérifications à l'aide d'un modèle numérique.

La modélisation est réalisée à l'aide d'un logiciel de calcul par éléments finis. Ce logiciel permet de suivre l'évolution des fissures jusqu'à la ruine de la structure. De nombreuses approches classiques sous forme de facteurs d'intensité $k$ [5], d'intégrale $J[6]$ ont déjà été réalisées. Pour notre part, les méthodes basées sur l'énergie de restitution $G[7]$ sont utilisées.

Dans le cadre du calcul non-linéaire, les lois de comportement les plus fidèles des différents matériaux doivent être prises en compte. Ces lois de comportement traduisent l'évolution des matériaux.

Ces lois sont de plus limitées en déformation. Les modèles simulés ont été maillés de façon homogène avec des éléments triangulaires physiquement non-linéaires.

Dans la première partie de notre travail, nous avons réalisé une série d'essais expérimentaux [8] afin de déterminer les lois de comportement des différents matériaux.

Dans la seconde partie, des simulations numériques ont été effectuées, en utilisant le code de calcul aux éléments finis URUS [9] sur un ovoïde témoin non renforcé et un ovoïde renforcé à l'aide de lamelles composites.

\section{Analyse non-linéaire par éléments finis}

Plusieurs méthodes analytiques $[10,11]$ ont été développées pour prédire le comportement en flexion d'éléments renforcés par collage de composites. Compte tenu des limites imposées par ces méthodes numériques, en raison notamment de la répartition complexe des contraintes dans le film de colle, il apparaît nécessaire de mettre en œuvre une méthode plus précise.

Cette méthode peut être fondée sur une discrétisation de la structure par éléments finis. Elle consiste à intégrer un modèle rhéologique du complexe béton/colle/composite en prenant en compte certaines de ces particularités, telles que la fissuration, le comportement non-linéaire de l'acier et du béton ou encore dans notre cas, les caractéristiques du film de colle.

La méthode des éléments finis est sans conteste la méthode d'analyse des structures la plus générale et la plus puissante. Elle permet la résolution approchée de nombreux problèmes de mécanique notamment en comportement non-linéaire. Cette approche suppose la prise en compte d'hypothèses fiables.

\section{Description du logiciel utilisé}

De nombreuses études sur les méthodes numériques $[12,13]$ ont déjà été réalisées depuis ces dernières années dans le domaine du béton. Cependant, la modélisation actuelle des ouvrages souffre de nombreux 
handicaps. Les structures sont généralement considérées comme élastiques non-linéaires, élasto-plastiques ou élasto-fragiles.

Il est possible avec les logiciels actuellement disponibles de représenter l'état des structures sous chargement.

Nous proposons de réétudier le problème dans le cadre de la mécanique de la rupture car les approches classiques sous forme de facteurs d'intensité des contraintes $k$ [5], d'intégrales de contour $J[6]$ ont été infructueuses. Récemment, un nouveau logiciel a été élaboré en utilisant les méthodes des taux de restitution d'énergie [7], taux de restitution permettant le calcul pour le béton.

Le logiciel d'éléments finis URUS version 8.2 [9] sera utilisé dans le cadre de cette étude.

La modélisation concerne les ovoïdes couramment rencontrés dans les réseaux d'assainissement. Le problème est traité en comportement non-linéaire avec fissuration. Les éléments choisis pour la modélisation sont des éléments à 3 nœuds (éléments surfaciques).

Les éléments finis utilisés sont des éléments plaques en membranes triangulaires ou quadrangulaires. Seuls les effets de membranes seront pris en compte (en état de contraintes planes). Mais l'élément est découpé en feuillets pour l'évaluation de l'énergie de restitution. La fissuration sera donc différente suivant qu'on est sur la peau ou à l'intérieur de l'élément. Par ailleurs, sur un même élément, le comportement est moyenné autour des points d'intégration («points de Gauss »). La mécanique de la rupture sera utilisée afin de discrétiser par élément et de calculer par feuillet (plaque) ou cellule (modèle de poutre). Lors de nos simulations, une résolution en élasticité non-linéaire sera utilisée.

\section{Hypothèses pour la modélisation}

\subsection{Hypothèses relatives à la modélisation}

Les paramètres relatifs à la modélisation sont la nature et les caractéristiques du sol, les charges appliquées et les conditions aux limites.

Notre modélisation concerne un ovoïde T180. Ce cas fait partie d'une étude de réhabilitation réalisée au sein du Bureau d'Études Structure \& Réhabilitation [14].

Les caractéristiques mécaniques du sol doivent être prises en compte sans négliger l'interface sol/structure. En effet, c'est cette interface qui permet de transmettre les sollicitations qui se produisent à l'intérieur de l'ouvrage vers le sol encaissant. Ce sol est un remblai limoneux correspondant au groupe de sol 4 d'après la classification RTR (Recommandations pour les terrassements routiers). Cette classification permet de regrouper les sols selon leurs caractéristiques géotechniques. Le coefficient de pression horizontale des terres est donc pour ce groupe $k=0$ (Fascicule 70). Ce cas est défavorable pour la fissuration en clef lors de surcharges verticales (deuxième cas de charge). Lors de cette étude, ni vides ni décompression ne sont pris au niveau de l'interface sol/structure.
Afin de se placer dans des conditions optimales de modélisation, nous avons remplacé le sol par une seule charge verticale appliquée sur le haut de l'ouvrage, le coefficient de pression des terres horizontale étant nul. Les charges sont appliquées par l'intermédiaire d'éléments « barres » liaisonnées directement sur l'extrados de l'ouvrage.

Afin que la ruine se produise bien dans les éléments représentant l'ouvrage, les éléments « barres », ont un module d'élasticité très faible et une résistance à la compression et en traction très élevée. La valeur de la charge appliquée est fonction de la couverture. Nous avons donc calculé la charge à appliquer en clef de voûte. Cette charge a été appliquée entre le nœud situé en clé de voûte et le noud situé en milieu de piédroit.

Les conditions aux limites ont été appliquées au niveau des nouds représentant l'axe de symétrie vertical de l'ouvrage et au niveau des nœuds situés à la base de l'ouvrage en extrados. Pour les nœuds situés sur l'axe de symétrie, nous avons laissé libre le déplacement suivant l'axe $Z$. Pour les nœuds situés à l'extrados en base de l'ouvrage, nous avons effectué un blocage complet des nœuds. L'ouvrage est appuyé sur le sol selon un angle d'appui $\alpha=60^{\circ}$.

\subsection{Modèle choisi}

La modélisation par éléments finis à l'aide du logiciel URUS [9] permet de prévoir le comportement des ouvrages renforcés ou non renforcés et d'obtenir l'apport du renfort.

Le type d'éléments finis choisis pour modéliser l'ouvrage est l'élément fini triangulaire physiquement nonlinéaire. Ces éléments ont la particularité d'avoir une non-linéarité comportementale. Seule la rigidité des éléments évolue selon le niveau de contraintes.

Le modèle choisi est maillé de façon homogène physiquement. Le pas du maillage a été affiné au cours des diverses simulations. Le pas retenu est un pas de 0,01 m. Pour les ovoïdes témoins, non renforcés, 502 éléments ont été nécessaires à la réalisation du modèle. Pour les ovoïdes renforcés, 510 éléments ont été nécessaires à la réalisation du modèle, 498 pour la structure béton, 6 pour la colle et 6 pour les lamelles. L'assemblage des différents matériaux de constitution des ovoïdes renforcés a été réalisé en liant les éléments avec des nœuds dont les degrés de liberté sont restés libres. La figure 2 représente le modèle d'un ovoïde T180 chargé verticalement.

\subsection{Caractéristiques et lois de comportement des différents matériaux}

Pour le support béton et le renfort composite, les lois de comportement ont été représentées en utilisant la loi de type «polyligne» incluse dans le logiciel URUS. En ce qui concerne l'adhésif, une loi de type « exponentielle »a été prise. Des essais expérimentaux ont été effectués afin 


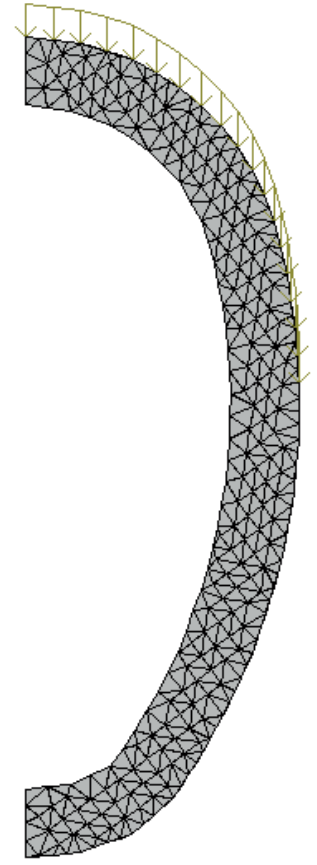

Fig. 2. Maillage d'un ovoïde T180.

de déterminer les caractéristiques des différents matériaux nécessaires à la détermination des lois de comportement.

Lors de nos simulations, les paramètres pris en compte pour les matériaux sont :

- Pour le béton :

Le comportement supposé isotrope du béton est décrit par son module $E=30000 \mathrm{MPa}$ et son coefficient de Poisson $\nu=0,2$.

- Pour le composite

Le comportement des lamelles de carbone est orthotrope. Cependant, pour nos calculs, seul la rigidité intervenant dans le sens des fibres est importante. Le module Ex est de $16500 \mathrm{MPa}$. Concernant les autres caractéristiques du composite unidirectionnel, elles sont prises nulles. En effet, ces caractéristiques sont très faibles vis-à-vis du haut module Ex. Les lamelles de carbone n'ont aucune résistance dans le sens perpendiculaire des fibres. De plus, au vu du comportement mécanique de l'ovoïde sous chargement vertical, seule la section transversale de l'ouvrage est soumise à de fortes contraintes, section correspondante au sens des fibres.

\subsubsection{Le béton}

Des essais de compression à 28 jours ont été réalisés sur trois lots de trois éprouvettes $16 \times 32$. Les éprouvettes ont été confectionnées à partir de la composition utilisée en usine de fabrication des ovoïdes T180 testés dans la suite de notre étude.

La résistance moyenne à la compression du béton à 28 jours est de 45,3 MPa. La résistance moyenne du béton

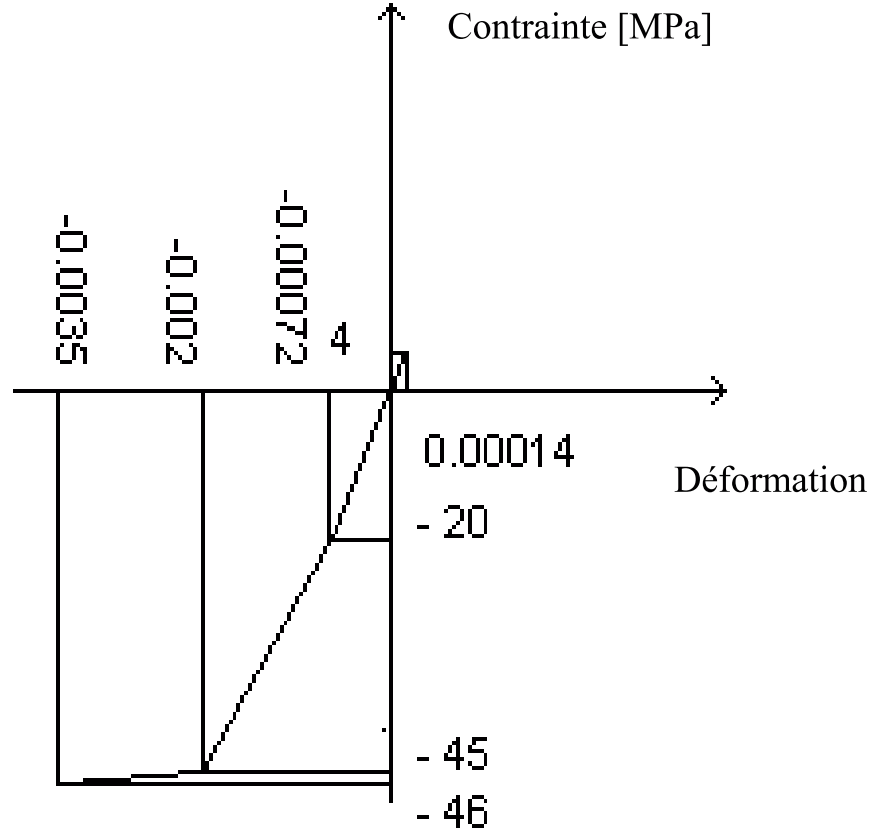

Fig. 3. Loi du comportement du béton.

à la traction est alors de 3,4 MPa. Le module de Young du béton a également été mesuré. Il est égal à $29300 \mathrm{MPa}$.

Ces différents essais effectués sur le support permettent de déduire alors la loi de comportement du béton. Une forme générale [15] des lois de comportement uni axiales retenues pour les milieux continus applicables au béton est indiquée figure 3 .

En ce qui concerne le renforcement, notre choix s'est porté sur des lamelles de carbone possédant un module de Young élevé. Ce type de renfort est très bien adapté aux ouvrages soumis à de la flexion.

\subsubsection{Les lamelles de carbone Sika Carbodur}

Les renforts composites se présentent sous la forme de lamelles de carbone (Sika Carbodur) [16] d'épaisseur 1,2 $\mathrm{mm}$ et de $80 \mathrm{~mm}$ de largeur. Ce sont des composites unidirectionnels pour lesquels la direction des sollicitations doit correspondre à celle des fibres (sens longitudinal). Les fibres de carbone agglomérées dans une matrice de résine époxydique représentent $60 \%$ du volume. Ces lamelles sont stockées en rouleaux et découpées sur chantier à la longueur désirée.

Les lois de comportement des lamelles de carbone ont été déduites d'études réalisées par traction simple [17]. Ces essais de traction directe réalisés sur les lamelles ont montré un comportement élastique linéaire jusqu'à la rupture.

Les résistances mécaniques du béton sont inférieures à celles du composite. Ce dernier matériau ne sera jamais sollicité jusqu'à la ruine. La loi de comportement est gardée linéaire (Fig. 4). 


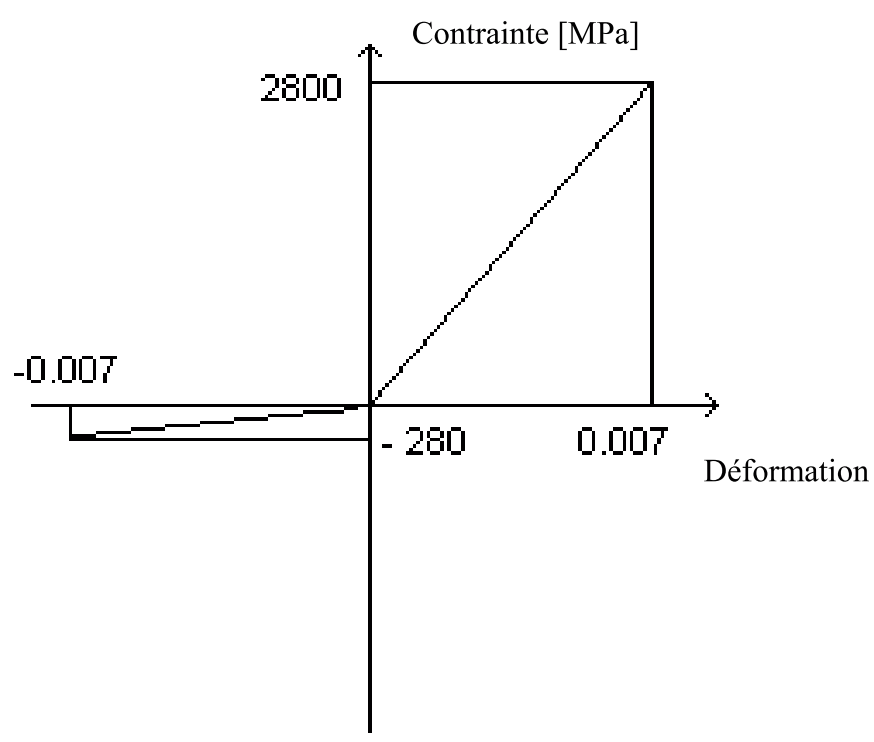

Fig. 4. Loi de comportement du composite.

\subsubsection{Le matériau de liaison : la colle}

C'est la Sikadur 30 Colle qui a été retenue pour le collage. Elle a été choisie pour sa conformité à la norme NF P 18-870 [18] sur les produits de collage. Cette colle est couramment employée sur les chantiers où elle a donné de bons résultats.

Le rôle de l'adhésif est très important dans le cas de renforcement par plats collés. En effet, il possède un double rôle qui consiste d'une part à maintenir le renfort sur le support béton et d'autre part à assurer la transmission des efforts exclusivement par cisaillement.

D'après Deuring [19], le comportement du film de colle se décompose en deux régions :

- Une région élastique linéaire délimitée par un glissement $\gamma_{\text {lin }}=0,011$ et par une contrainte $\tau_{\text {lin }}=$ 31,87 N.mm ${ }^{-2}$. Pour cette première région, il est possible de définir un module de glissement $G$, égal pour ces essais à $G=2962 \mathrm{~N} . \mathrm{mm}^{-2}$.

- Une région plastique qui s'étend de $\gamma_{\text {lin }}$ et $\gamma_{\max }=$ 0,018 et de $\tau_{\text {lin }}$ à $\tau_{\max }=32,40 \mathrm{~N} \cdot \mathrm{mm}^{-2}$ pour laquelle il est également possible de définir un modèle de glissement $G^{\prime}=75,7$ N.mm ${ }^{-2}$.

Les caractéristiques mécaniques de cet adhésif sont décrites dans le tableau 1. Le logiciel de calcul des structures URUS possède un « catalogue » de lois prédéfinies. Les lois de comportements alors adoptées pour l'adhésif sont de type exponentiel. Ce type de loi permet de reproduire la région élastique linéaire puis la région plastique (Fig. 5).

\section{Resultats de la modélisation d'ovoïdes}

Pour notre modélisation, nous avons modélisé un ovoïde (témoin) en béton T180 soumis à un chargement correspondant à 2 mètres de remblais de sol limoneux.

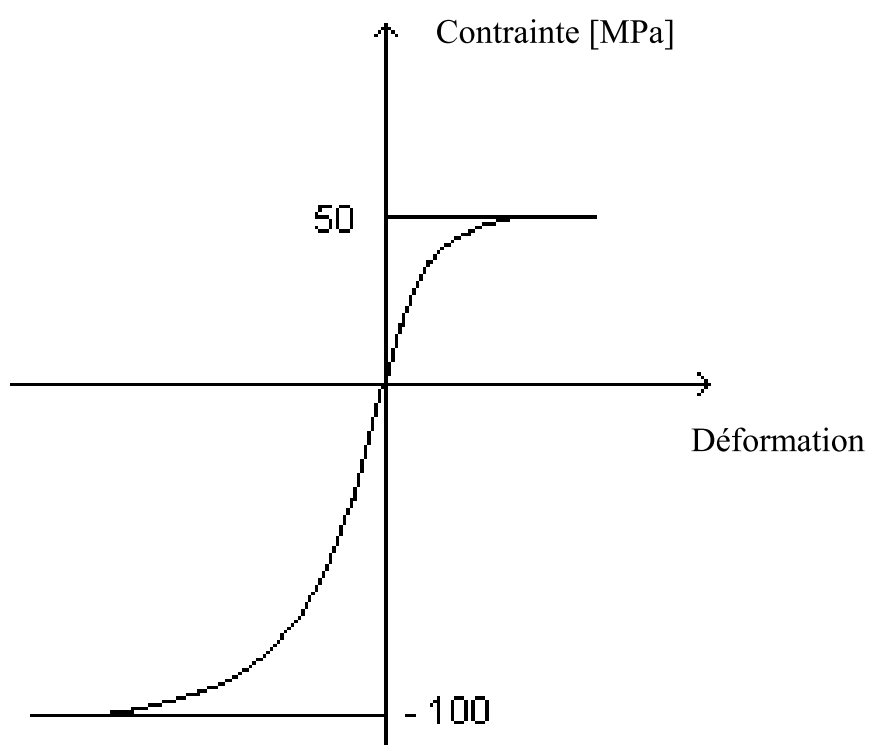

Fig. 5. Loi de comportement de la colle.

Tableau 1. Caractéristiques mécaniques de l'adhésif.

\begin{tabular}{lc}
\hline & Sikadur 30 Colle \\
\hline Résistance à la compression [MPa] & 100 \\
Résistance à la traction [MPa] & 50 \\
Module d'élasticité [MPa] & 12800 \\
Durée pratique d'utilisation à $20{ }^{\circ} \mathrm{C}$ & 1 heure 10 \\
\hline
\end{tabular}

Une série d'incrémentation de charge a été réalisée afin d'obtenir la ruine complète de l'ouvrage.

Le mécanisme de ruine de l'ovoïde étant connu, la mise en place de plats composites dans les parties les plus sollicitées a été effectuée. Une fois le complexe colle-lamelles de carbones positionnés sur le support béton, le calcul est mené jusqu'à la non-convergence du processus itératif. Les résultats de cette seconde modélisation permettent d'une part d'obtenir le gain de résistance et d'autre part d'observer si une redistribution des contraintes est présente.

\subsection{Ovoïde témoin}

La charge initiale correspond à une hauteur de remblai de 2,00 mètres. Nous avons incrémenté cette charge jusqu'à non convergence du processus de calcul. La ruine s'est produite au $28^{\mathrm{e}}$ pas d'incrémentation soit 2,8 fois la valeur de la charge initiale, soit environ une charge correspondant à 6 mètres de remblai.

Le mécanisme de ruine de l'ouvrage correspond aux mécanismes de déformations obtenues lors de la réalisation de calculs théoriques basés sur les lois fondamentales de la résistance des matériaux d'ovoïdes en béton [20].

La fissuration intervient à l'intrados en clef, à l'extrados en piédroits et à l'intrados au niveau de la liaison radier-piédroits. La zone la plus fissurée se situe en clef de l'ouvrage.

Les contraintes maximales relevées lors de la rupture sont données figure 6 . 


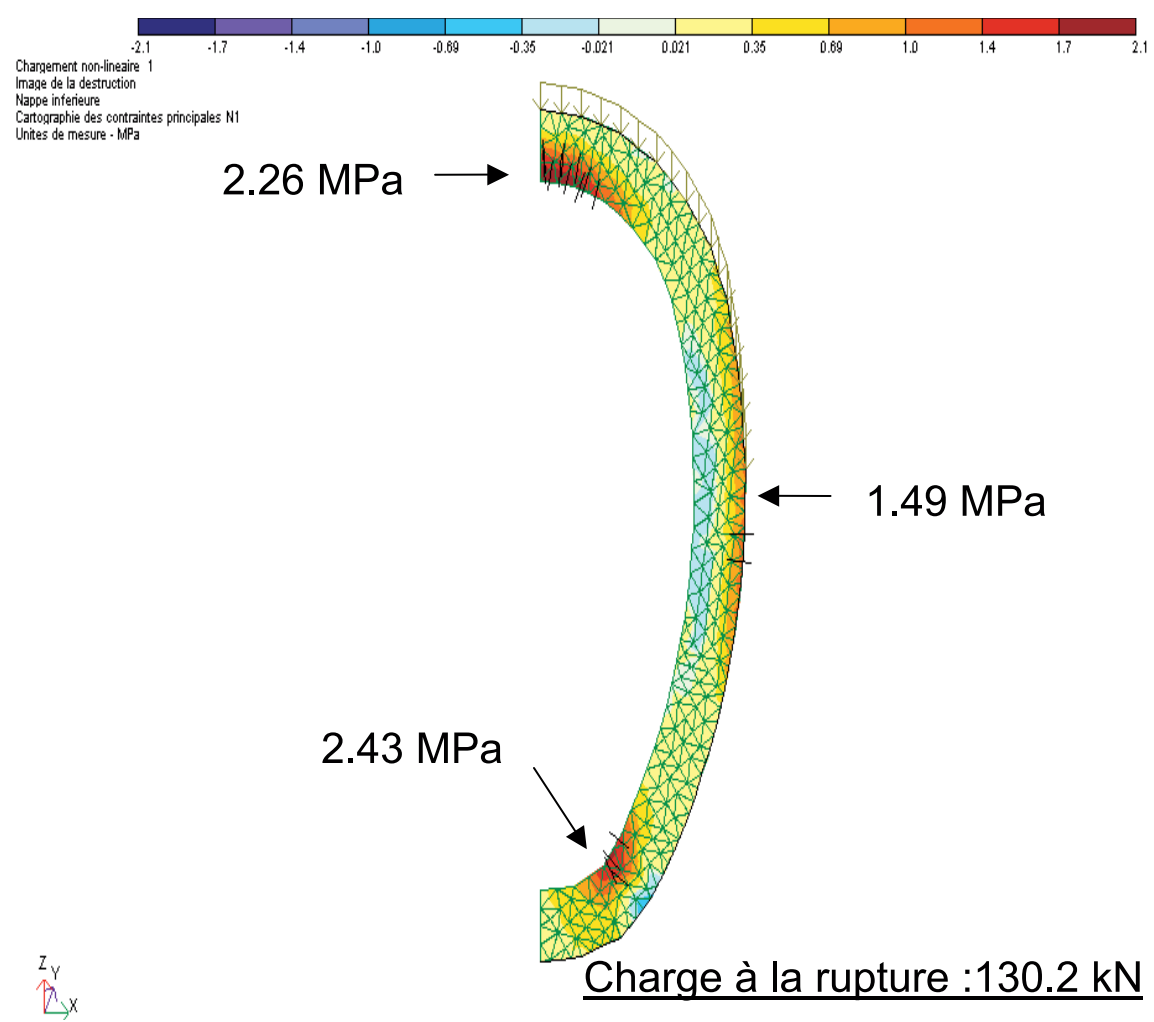

Fig. 6. Mécanisme de ruine de l'ovoïde témoin.

Les différentes valeurs sont détaillées selon les trois zones décrites ci-dessus.

\section{Au niveau de la clef de voûte :}

La valeur maximale de la contrainte principale N1 est de 2,26 MPa. Les fissures sont situées à l'intrados en clef de l'ouvrage, elles se propagent de la zone la plus tendue vers la zone comprimée du béton. De plus, la contrainte de traction présente en clef est de 1,70 MPa.

\section{Au niveau du milieu de piédroits :}

La valeur maximale de la contrainte principale relevée en milieu de piédroits est égale à 1,49 $\mathrm{MPa}$. La contrainte de traction est quant à elle égale à 1,50 MPa. La zone tendue pour cette partie de l'ouvrage se situe à l'extrados. Les valeurs des contraintes de traction n'indiquent pas la ruine de l'ouvrage. Cependant, il est à noter la présence de quelques fissures dans cette zone tendue située à l'extrados de l'ouvrage

\section{Au niveau du bas de piédroits (liaison radier-piédroits) :}

Pour cette portion de l'ouvrage, nous obtenons une valeur maximale de la contrainte principale N1 de 2,43 MPa. Cette valeur correspond à la valeur maximale de la contrainte présente dans l'ouvrage. La ruine s'est donc produite à ce niveau. La valeur de la contrainte de traction est de 1,38 MPa.

$\mathrm{Au}$ vu de ces différentes valeurs, la ruine de l'ouvrage s'est produite au niveau de la clef de voûte.

\subsection{Ovoïde renforcé par lamelle de carbone}

Avant un renforcement par pose de lamelles de carbone, il sera préalablement procédé au traitement des fissures, avec leurs étanchements éventuels. La réfection du radier devra être effectuée comme lors d'une restructuration par chemisage en béton projeté [2].

La zone de renfort, au vu de la ruine de l'ouvrage non renforcé, sera la clef de voûte. Nous avons donc procédé à l'optimisation de cette zone.

Lors de nos simulations, nous sommes partis d'une surface de $1 \mathrm{~m}^{2}$ à renforcer puis nous l'avons réduite. Nos critères pour déterminer cette surface sont au nombre de deux :

- augmentation de charge à la rupture,

- baisse significative des contraintes présentes dans l'ouvrage.

La surface de renforcement a été obtenue par processus heuristique en optimisant la surface de renforcement. Au fur et à mesure des calculs, la zone de renforcement s'est vue diminuée en respectant les critères de détermination de cette surface.

Les lamelles sont disposées dans le sens transversal de l'ouvrage. En effet, les lamelles utilisées sont des lamelles unidirectionnelles. Les fibres sont disposées dans le sens longitudinal de la lamelle.

La surface à renforcer s'étend sur les trois premiers éléments modélisés sur le demi-ouvrage ce qui représente une surface totale de $0,75 \mathrm{~m}^{2}$ par mètre linéaire d'ouvrage. 


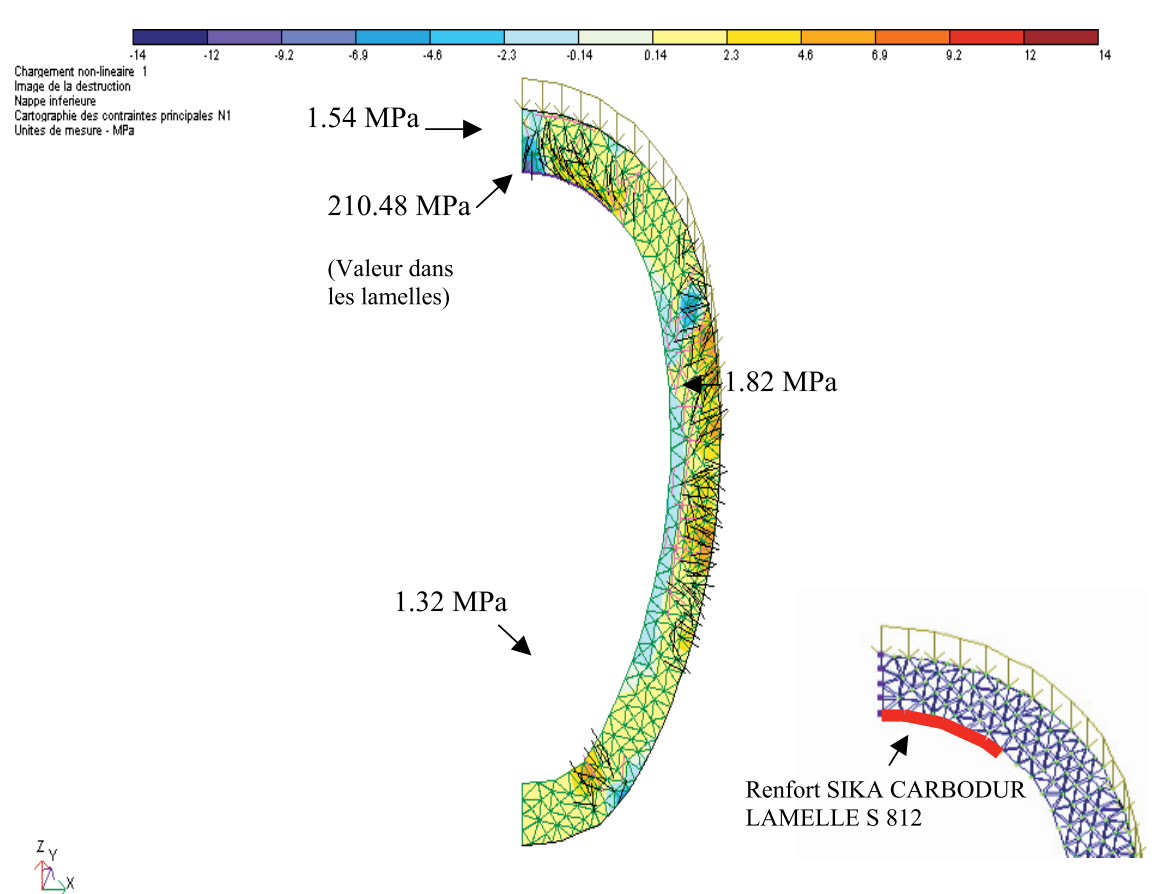

Fig. 7. Mécanisme de ruine de l'ovoïde renforcé.

Lors de la simulation de l'ouvrage renforcé sur les deux premiers éléments du demi-ouvrage (soit $0,50 \mathrm{~m}^{2}$ de renforts par mètre linéaire d'ouvrage), nous avons une charge de rupture proche de celle obtenue lors du renforcement sur les trois premiers éléments (soit $0,75 \mathrm{~m}^{2}$ de renforts par mètre linéaire d'ouvrages) mais les contraintes présentes dans l'ouvrage restent élevées.

Le mécanisme de ruine de l'ouvrage est représenté figure 7, nous avons décidé de montrer les fissures dans la nappe inférieure sur la cartographie des contraintes principales N1.

Les fissures se situent principalement à l'intrados en clef et à l'extrados en piédroits. Quelques fissures apparaissent au niveau de la liaison radier-piédroits.

\section{Au niveau de la clef :}

Le mécanisme de ruine de la clef est représenté figure 8. La ruine de l'ouvrage s'est produite au $42^{\mathrm{e}}$ pas d'incrémentation, soit à 4,2 fois la charge appliquée.

Les fissures se propagent dans l'ouvrage de l'intrados vers l'extrados. Les lamelles ne sont pas altérées lors du chargement de l'ouvrage. Les valeurs des contraintes obtenues en clef sont données figure 8 .

La contrainte de traction présente dans l'ouvrage est inférieure à la contrainte admissible. Lors de la modélisation du modèle non renforcé, la contrainte de traction était de 1,70 MPa pour l'application de la seule charge. Ici, nous obtenons 1,54 MPa pour cette même contrainte mais pour 4,2 fois la charge. Les lamelles ont donc absorbé les efforts présents en clef.

La contrainte de traction obtenue dans la lamelle est nettement inférieure à la contrainte admissible. Cette contrainte maximale se situe sur l'élément situé en clef de voûte. La clef est donc la zone la plus tendue de l'ouvrage. Au niveau de la colle, la contrainte la plus élevée est repérée sur le dernier élément renforcé. Cependant les contraintes dans la colle restent faibles par rapport aux contraintes admissibles. La contrainte de cisaillement relevée est de 2,93 $\mathrm{MPa}$. La région élastique de la colle est délimitée par une contrainte admissible de 31,87 MPa. Les contraintes présentent dans les lamelles de carbone n'excédent pas la valeur admissible. La contrainte moyenne de traction est de 210,48 MPa au sein du renfort.

Compte tenu des résultats obtenus en clef, le renforcement de l'ouvrage ovoïde par collage de lamelles de carbone est concluant. Cependant, nous allons observer si le fait de renforcer l'ouvrage en clef n'a pas de conséquence défavorable sur la redistribution des contraintes sur le reste de l'ouvrage.

\section{Au niveau du milieu de piédroit :}

Le mécanisme de ruine du milieu de piédroits est représenté figure 9 . La zone tendue du béton se situe à l'extrados pour les piédroits. Les fissures se propagent donc de l'extrados vers l'intrados de l'ouvrage.

Par comparaison aux contraintes obtenues sur l'ouvrage non renforcé, nous avons une augmentation sensible de la contrainte de traction. Cette augmentation est de l'ordre de $20 \%$. Cependant, la répartition des contraintes ne se fait plus de façon uniforme mais on peut observer sur la cartographie des contraintes des zones où les contraintes sont élevées. Cette augmentation est le résultat de l'accroissement des charges. La contrainte principale $\mathrm{N} 1$ a augmenté considérablement, elle passe de 1,82 $\mathrm{MPa}$ au niveau de l'intrados à $13,44 \mathrm{MPa}$ à l'extrados.

Les fissures dans le support béton sont plus nombreuses que dans le cas de l'ovoïde non renforcé, ce qui 


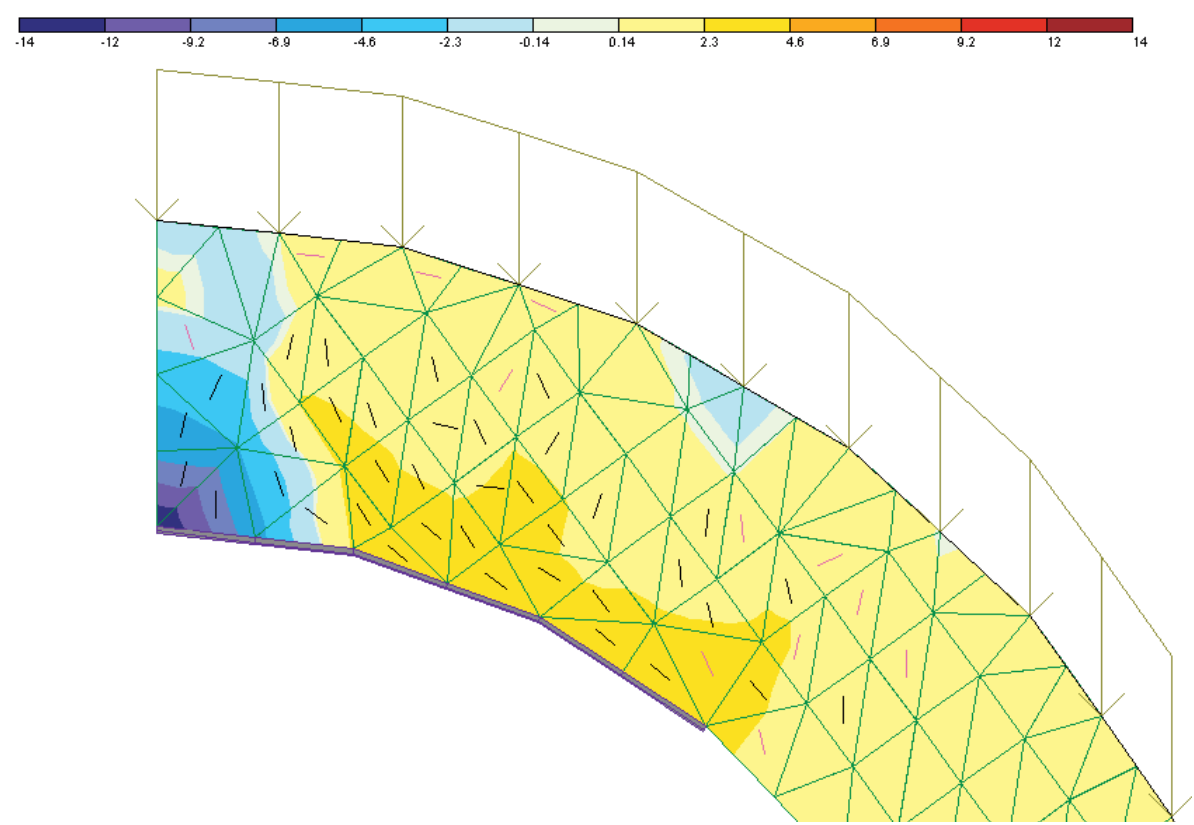

Fig. 8. Détail de la ruine au niveau de la clé.

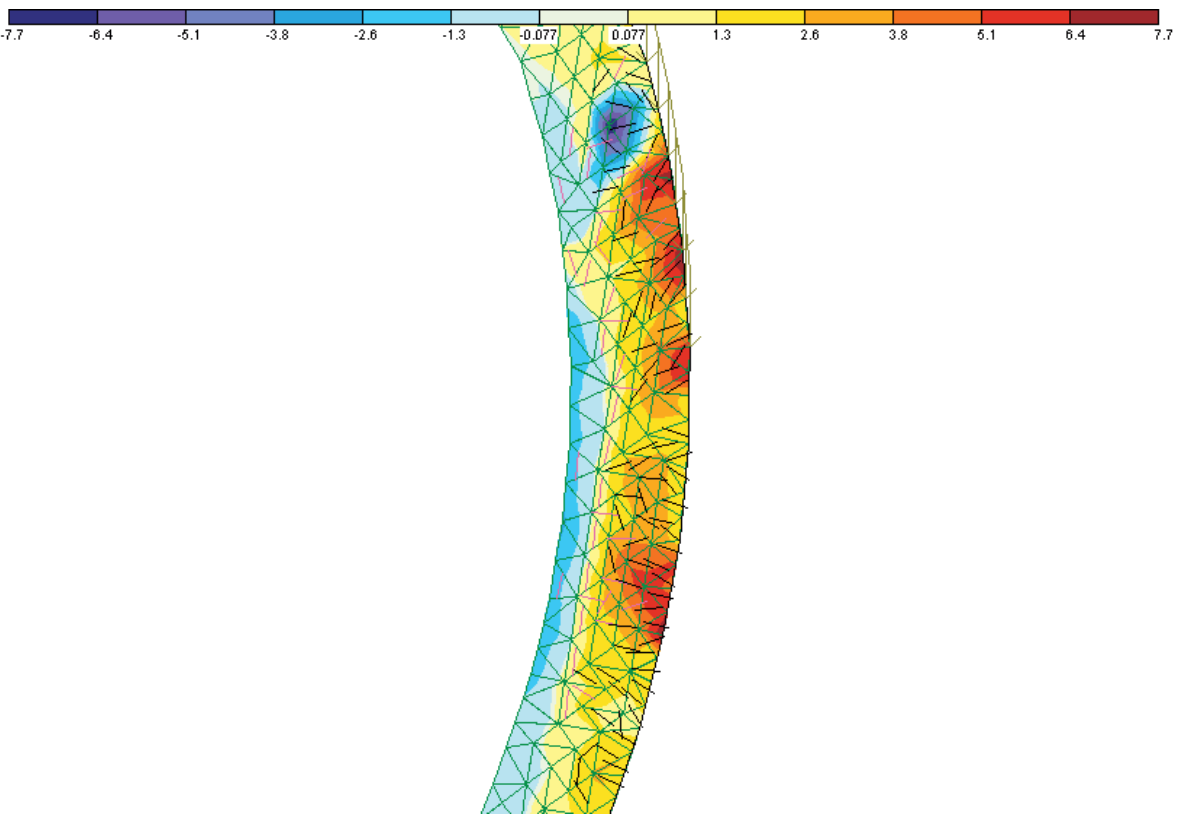

Fig. 9. Détail de la ruine au niveau du milieu de piédroit.

est normal au vu des contraintes présentes et de la charge appliquée lors de la ruine de l'ouvrage.

Les contraintes ont donc été redistribuées dans le piédroit.

\section{Au niveau du bas de piédroits (liaison radier-piédroit) :}

Le mécanisme de ruine de la partie inférieure de l'ouvrage est représenté figure 10. La cartographie des contraintes principales N1 montre une légère zone de désordres au niveau de la liaison radier-piédroit. Cette zone de liaison est marquée par des fissures se propageant de l'intrados de l'ouvrage vers l'extrados. De plus, nous remarquons une zone de compression située au niveau du dernier appui entre l'ouvrage et le sol.

Par rapport à l'ouvrage non renforcé, la valeur de la contrainte de traction en bas de piédroits a sensiblement diminué. La contrainte de traction est de 1,32 $\mathrm{MPa}$ au niveau de la liaison radier/piédroit.

Nous pouvons donc conclure à une redistribution des contraintes lors de la mise en place d'un renforcement. Les contraintes ont diminué en clef et en radier de l'ovoïde, 


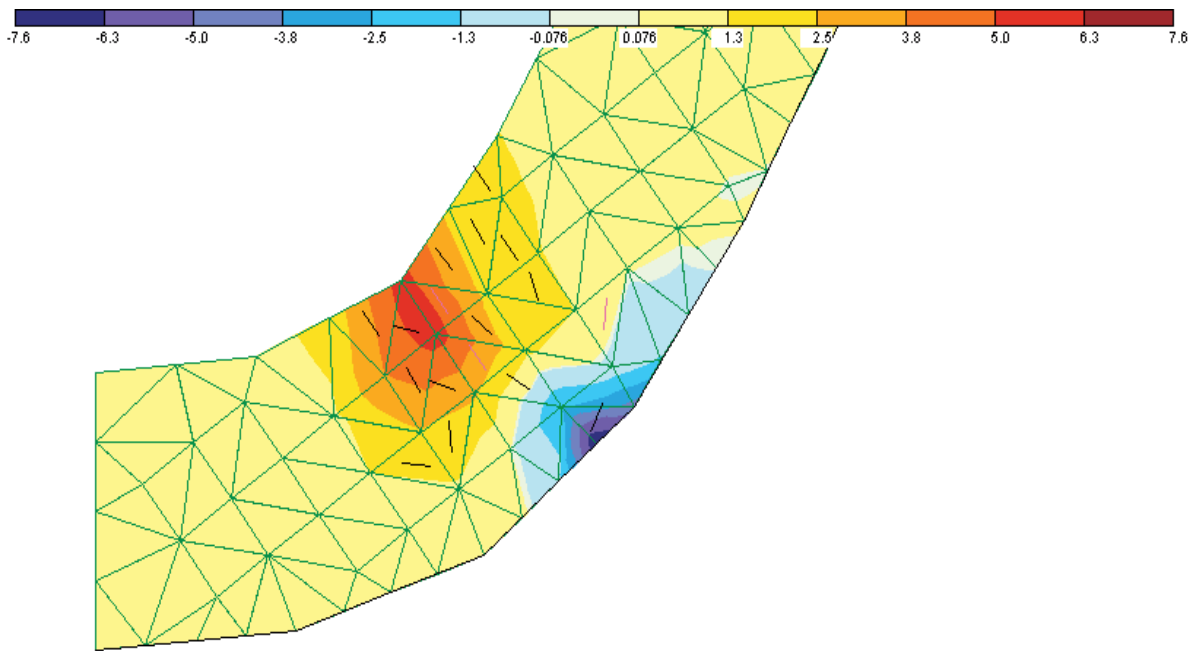

Fig. 10. Détail de la ruine au niveau du radier.

mais elles ont augmenté en piédroits. Le fait de coller des lamelles de carbone a donc permis de diminuer les contraintes dans l'ouvrage. L'augmentation remarquée en piédroits se situe à l'extrados de l'ouvrage. Dans la réalité, l'ouvrage sera «bloqué » latéralement par le sol encaissant l'ouvrage.

\section{Conclusion}

Le calcul non-linéaire a permis de visualiser les différentes zones de fissuration et d'aller jusqu'à la ruine de l'ouvrage.

Les résultats de la pré-modélisation sont les suivants :

- examen de l'application d'un renforcement ponctuel lors de surcharges verticales;

- optimisation des zones de renforcement selon le type de collecteur.

Le renforcement pour une surcharge verticale montre un gain important. De plus, malgré un chargement plus important, les contraintes de traction dans les zones sensibles ont diminué. Cela signifie que les renforts ont absorbé les charges appliquées et que nous avons une redistribution des contraintes vers le milieu de piédroits à l'extrados. Cependant, cette augmentation de contrainte en milieu de piédroits n'est pas significative du fait de la reprise de ces contraintes par le sol environnent.

Dans ces conditions de réparation, la solution de réhabilitation ponctuelle est de $55 \%$ moins coûteuse que dans le cas d'une réhabilitation ponctuelle.

Remerciements. Nous tenons à remercier les sociétés SIKA France, STRUCTURE \& REHABILITATION et VALENTIN Environnement et Travaux Publics pour leur partenariat et leur soutien financier dans la réalisation du projet.

\section{Références}

[1] Projet National RERAU, Restructuration des collecteurs visitables, Guide technique, Lavoisier, 2002, pp. 1-2

[2] S. Kesteloot, V. Hembise, J. Lefort, Pathologies et méthodes de réparation des ouvrages d'assainissement visitables - Évaluation des coûts, Projet de Recherche de Maîtrise, Faculté des Sciences Appliquées, Béthune, 2000

[3] Formation de l'H2S dans les réseaux d'assainissement, conséquences et remèdes, Synthèse Bibliographique, Institut de Recherche Hydrologiques de Nancy, 1987

[4] C. Djelal, E. David, F. Buyle-Bodin, Y. Giquel, Renforcement des poutres en béton armé à l'aide de lamelles composites : Étude expérimentale Prédimentionnement, Annales du bâtiment et des travaux publics 1 (1998) 39-47

[5] G.R. Irwin, Analysis of stresses and strains near the end of a crack traversing a plate, Trans. ASME, J. Appl. Mech. E24 (1957) 361

[6] J.R. Rice, A path independent integral and approximate analysis of strain concentration by notches and cracks, J. Appl. Mech. 35 (1968) 379-386

[7] J. Lemaître, J.L. Chaboche, Mécanique des matériaux solides, Dunod, Paris, France 1985

[8] S. Kesteloot, Étude expérimentale des liaisons béton fissuré - lamelles/Résultats des essais de collages, Rapport de première année de thèse, Faculté des Sciences Appliquées, Béthune, 2002

[9] URUS Version 8.2, Logiciel de calcul de structures par éléments finis, @bd soft

[10] B. Täljsten, Plate bonding, strengthening of concrete structures with epoxy bonded plates of steel or fiber reinforced plastics, Doctoral thesis, Lulea University of Technology, 1994

[11] T.C. Triantafillou, Shear strengthening of reinforced concrete beams using epoxy bonded FRP composite, ACI Structural J. 2 (1995) 107-115

[12] M. Arduini, A. Di Tommaso, O. Manfroni, Fracture mechanics of concrete beams bonded with composites plates, Non-metallic (FRP) Reinforcement for concrete structures, L. Taerwe (ed.), RILEM (1995) 485-491 
[13] T.C. Triantafillou, N. Deskovic, M. Deuring, Strengthening of concrete structures with prestressed fiber reinforced plastic sheets, ACI Structural J. 89 (1992) 235-244

[14] Étude de diagnostic et préconisation de travaux de réhabilitation, commune de Nogent sur Marne, Structure \& Réhabilitation, Affaire $\mathrm{n}^{\circ}$ 806, 2001

[15] J. Mazars, Mécanismes physiques de rupture et modèles de comportement du béton hydraulique in « Le béton hydraulique », Presse de l'École Nationale des Ponts et Chaussées, Paris, 1982

[16] SIKA - Procédés de renforcement des structures par collage d'armatures à base de fibres de carbones, Cahier des clauses techniques CCT 37, août 2000
[17] E. David, Comportement mécanique de poutres en béton armé renforcées ou réparées par collage de matériaux composites - Étude expérimentale et modélisation, Thèse de doctorat, Université d'Artois, 1999 pp. 85-90

[18] Norme NF P 18-870, Produits ou systèmes de produits à base de résines synthétiques ou de liants hydrauliques pour collage structural entre deux éléments en béton Caractères normalisés garantis, AFNOR, 1993

[19] M. Deuring, Verstärken von Stahlbeton mit gespannten Faserverbundwerkstoffen, Thèse de doctorat, Eidgenössischen Technischen Hochschule, Zürich, 1993

[20] A. Guerrin, G. Daniel, Traité de béton armé - Ouvrages enterrés, Dunod, 1969 